Revista de Biología Marina y Oceanografía

Vol. 49, №2: 157-173, agosto 2014

DOI 10.4067/S0718-19572014000200001

\title{
REVISIÓN
}

\section{Microalgas, cultivo y beneficios}

\author{
Microalgae, culture and benefits
}

\author{
Alexis Hernández-Pérez ${ }^{1}$ y José I. Labbé ${ }^{1}$ \\ ProCycla, General Eugenio Garzón 6561, Vitacura, Región Metropolitana, Chile. ahern@procycla.com, nlabbe@procycla.com
}

\begin{abstract}
Microalgae have a phycoremediation capacity through the removal or biotransformation of pollutants from a liquid or gaseous medium. These pollutants are captured by the growing algal biomass allowing them to be recovered through harvest. This capability results in a culture system with two purposes: removal of contaminants and biomass production for commercial uses. Both goals depend on the cultivation system, cultivated species and environmental factors. The use of contaminated media in culture impacts directly on production cost. Selecting the type of culture system is important and should be done based on biological, technical, environmental and economic criteria previously settled. Between several techniques differentiated in both efficiency and complexity, the algal biomass harvest is the most complex and expensive process in microalgae cultivation. Biomass production from microalgae has provided a wide range of biotechnological products, uses in the food industry, health and medicine, animal feed, organic compounds and biofuels production. All this is of great importance due to global environmental issues that exist today. This paper makes a summary and reviews the latest information (from 1972 to 2014) on different aspects and parameters of relevance for microalgae cultivation, its many uses and benefits.
\end{abstract}

Key words: Microalgae, phycoremediation, culture, harvest, biomass, nutrients, wastewater, biofuels

Resumen.- Las microalgas poseen una capacidad ficorremediadora que consiste en la eliminación o biotransformación de contaminantes de un medio líquido o gaseoso. Estos compuestos contaminantes son captados por la biomasa algal y pueden ser recuperados mediante su cosecha. Esta capacidad resulta en un sistema de cultivo con 2 propósitos: eliminación de contaminantes y producción de biomasa con fines comerciales. Ambos objetivos dependen del sistema de cultivo, la o las especies cultivadas y los factores ambientales. La utilización de medios contaminados en el cultivo impacta directamente en los costos de producción. La elección del tipo de sistema de cultivo es importante, y debe realizarse en base a factores biológicos, técnicos, ambientales y económicos, definidos previamente. La cosecha de la biomasa algal es el procedimiento más complejo y costoso en el cultivo de microalgas, existiendo varias técnicas diferentes tanto en eficiencia como en complejidad. La producción de biomasa de microalgas ha proporcionado una amplia gama de productos biotecnológicos con usos en la industria alimenticia, salud y medicina humana, alimentación animal, compuestos orgánicos y biocombustibles. Todo esto adquiere una gran importancia debido a los problemas ambientales globales existentes en la actualidad. El presente trabajo, recopila de forma general la información actualizada (1972 a 2014) sobre diferentes aspectos y parámetros de importancia en el cultivo de microalgas, sus múltiples usos y beneficios.

Palabras clave: Microalgas, ficorremediación, cultivo, cosecha, biomasa, nutrientes, aguas residuales, biocombustibles

\section{INTRODUCCIÓN}

Hoy en día es innegable la existencia de un cambio climático a nivel planetario (Brennan \& Owende 2010, Stager 2012). La magnitud de este fenómeno es tal, que forma parte de la agenda política de los estados y organismos internacionales (Chiu et al. 2011, Stager 2012). Cambio climático involucra, entre otros, sequías, inundaciones y/o acidificación de los océanos, a nivel local y global (Chen et al. 2011, Stager 2012), siendo la actividad humana la principal responsable, en especial, por la combustión de combustibles fósiles (Olguín 2003, Chen et al. 2011, Chiu et al. 2011, Stager 2012), impulsor del $80 \%$ de producción de energía (Chen et al. 2011).
El rápido desarrollo industrial impone un alto riesgo a la disponibilidad y calidad de las aguas (Dominic et al. 2009, Abdel-Raouf et al. 2012) y del aire, debido a la cantidad de productos contaminantes generados, como nutrientes y $\mathrm{CO}_{2}$ (Chiu et al. 2011, González-López et al. 2011, Abdel-Raouf et al. 2012).

La contaminación de los cuerpos de agua radica en la eutrofización, que es causada por la liberación de compuestos orgánicos e inorgánicos al medio (Lavoie \& de la Noüe 1985, Rawat et al. 2011) con concentraciones de nitrógeno y fósforo que pueden alcanzar hasta 3 veces o más de lo normal (Park et al. 2011a, Rawat et al. 2011), 
permitiendo la proliferación de microalgas dañinas para el hombre, lo que afecta la calidad de las aguas (McGriff \& McKinney 1972, Olguín 2003, León \& Chaves 2010).

Por otro lado, se estima que hacia el año 2300, el $\mathrm{CO}_{2}$ atmosférico alcanzará un máximo de 1900 a 2000 ppm, 5 veces mayor que el actual, generando en los océanos un solvente corrosivo para todos aquellos organismos con esqueleto carbonatado (Stager 2012).

En este contexto, el desarrollo de tecnologías orientadas al cuidado del medio ambiente, a la reutilización de los residuos y a la generación de energías limpias de bajo costo se ha tornado indispensable. Entre estas tecnologías, los cultivos de microalgas adquieren un gran protagonismo, al ser considerados eco-amigables (ecofriendly process), ya que reciclan eficientemente contaminantes desde medios líquidos y gaseosos (Olguín 2003, Dominic et al. 2009, Doušková et al. 2010, León \& Chaves 2010, González-López et al. 2011, Rawat et al. 2011), incorporándolos a su metabolismo para generación de biomasa (Markou \& Georgakakis 2011).

En comparación con otros sistemas de tratamientos de aguas, los cultivos de microalgas son considerados una tecnología de mediana complejidad, con desarrollo en escala de plantas pilotos (Flotats et al. 2011). Al tratarse de organismos vivos involucran una serie de parámetros (i.e., nutrientes, luz) que deben ser considerados, evaluados, determinados y medidos para realizar con éxito un cultivo. Además cambian óptimos según la especie cultivada (Grobbelaar 2004, Richmond 2004, Park et al. 2011a). Así mismo, los cultivos presentan una gran variedad de diseños, pudiéndose clasificar en 2 grandes grupos: sistemas abiertos y cerrados; cada uno con una variedad importante de diseños diferentes (Borowitzka 1999, Contreras-Flores et al. 2003, Tredici 2004). El tipo de cultivo y la especie a utilizar dependerá entre otros, del objetivo que se quiera conseguir.

Si bien la mediana complejidad de la ficorremediación, la convierte en una técnica atractiva como fuente de nutrientes y/o $\mathrm{CO}_{2}$, su asociación a procesos productivos de biomasa aún presenta una serie de dificultades que se deben mejorar, como la relación costo/eficiencia en los actuales sistemas de separación de la biomasa (cosecha) desde el medio líquido (Abeliovich 2004, Flotats et al. 2011, Rawat et al. 2011). La biomasa de microalgas presenta una gran variedad de productos con valor económico (Day et al. 2009, Hou et al. 2011), que al asociarse a la ficorremediación, pueden reducir los costos del cultivo (Rosales et al. 2007, Abdel-Raouf et al. 2012).
A continuación se recopila de forma general, la información más relevante actualizada y destacada entre los años 1972 y 2014, sobre microalgas en relación a sus capacidades en parámetros de cultivo, sistemas de cultivos, ficoremediación, cosecha y usos de la biomasa.

\section{Parámetros a considerar en un Sistema de cultivo}

\section{ESPECIES DE MICROALGAS}

La elección de las especies a cultivar depende directamente de la finalidad que se le desea brindar a la biomasa resultante (e.g., pigmentos, alimento) y/o si el cultivo es para ficorremediación. Las especies algales predominantes dentro de un sistema abierto dependen de factores ambientales, operacionales y parámetros biológicos (McGriff \& McKinney 1972, Park et al. 2011a, Abdel-Raouf et al. 2012). En un sistema cerrado se pueden lograr cultivos monoespecíficos aislados del medioambiente (Posten 2009).

Las microalgas en un cultivo para ficorremediación deben cumplir con 3 condiciones: alta tasa de crecimiento; alta tolerancia a la variación estacional y diurna si es un sistema abierto; y buena capacidad para formar agregados para una cosecha por simple gravedad (Park et al. 2011b). Además, altos niveles de componentes celulares valiosos (por ejemplo lípidos para generación de biodiesel) también podrían ser deseables (Martínez 2008, Park et al. 2011a, Abdel-Raouf et al. 2012).

Algunas especies presentes en aguas contaminadas son utilizadas en tratamientos de aguas residuales por su elevada tolerancia. Además, varias de éstas también son utilizadas para fines comerciales específicos (Park et al. 2011a, Park et al. 2011b, Abdel-Raouf et al. 2012). Los géneros Chlorella, Ankistrodesmus, Scenedesmus, Euglena, Chlamydomonas, Oscillatoria, Micractinium, Golenkinia, Phormidium, Botryococcus, Spirulina, Nitzschia, Navicula y Stigeoclonium han sido registrados en aguas residuales desde distintas procedencias (Borowitzka 1999, Rawat et al. 2011, AbdelRaouf et al. 2012). Varias de éstas son comercialmente interesantes para la alimentación humana y/o animal, la obtención de biocombustibles, aceites esenciales, pigmentos, entre otros usos (Borowitzka 1999, Harun et al. 2010).

\section{LuZ}

La intensidad lumínica es uno de los principales parámetros a considerar en un cultivo (Contreras-Flores 
et al. 2003). En ausencia de limitación por nutrientes, la fotosíntesis se incrementa con el aumento de la intensidad lumínica, hasta alcanzar la máxima tasa de crecimiento específica para cada especie en el punto de saturación por luz (Park et al. 2011a). Pasado este punto, se alcanza el punto de fotoinhibición, con resultados perjudiciales para la misma célula e incluso la muerte, implicando pérdida de eficiencia fotosintética y productividad del cultivo (Contreras-Flores et al. 2003, Richmond 2004, Martínez 2008, Park et al. 2011a).

Los cultivos microalgales exteriores suelen sufrir fotoinhibición en las principales horas del día debido a la alta intensidad lumínica (Martínez 2008). Bajo ciertas condiciones, los cultivos con mayor densidad celular (> $3 \mathrm{~g} \mathrm{~L}^{-1}$ ) son capaces de utilizar la luz incidente con mayor eficiencia en comparación con cultivos convencionales diluidos (Contreras-Flores et al. 2003). Esto se debe al autosombreado, donde las células más cercanas a la superficie dan sombra a las capas inferiores, con células más alejadas de la superficie (Contreras-Flores et al. 2003, Markou \& Georgakakis 2011, Park et al. 2011a). Un sistema de mezcla eficiente permitirá un acceso periódico de todas las células a la luz, de lo contrario, será perjudicial puesto que la intensidad lumínica decrece con el aumento de la turbidez (Contreras-Flores et al. 2003, Markou \& Georgakakis 2011). En sistemas HRAP (High Rate Algal Ponds), el mecanismo de paletas provee una eficiente mezcla vertical permitiendo una exposición intermitente de las células a la luz (Park et al. 2011a).

\section{Temperatura}

La producción algal aumenta proporcionalmente con la temperatura hasta alcanzar la temperatura óptima de cada especie. Por encima de esta, aumenta la respiración y la fotorrespiración reduce la productividad global. La temperatura óptima varía entre las especies, pero en general está entre $28^{\circ}$ y $35^{\circ} \mathrm{C}$ (Park et al. 2011a).

En un sistema de cultivo cerrado, la temperatura se puede controlar por varios mecanismos, tales como rociadores de agua, inmersión del colector solar en piscinas, reactor dentro de un invernadero, etc. (Martínez 2008). Por el contrario, en un sistema de cultivo abierto es muy difícil de controlar, aunque se pueden realizar ciertas acciones simples para disminuir el efecto, como cubrir los estanques con plásticos transparentes (Borowitzka 1999, Martínez 2008, Park et al. 2011a).

\section{${ }_{\mathrm{PH}} \mathrm{CO}_{2}$}

El pH del cultivo está influenciado por varios factores como la productividad algal, la respiración, la alcalinidad y composición iónica del medio de cultivo, la actividad microbiana autotrófica y heterotrófica y la eficiencia del sistema de adición de $\mathrm{CO}_{2}$ (Martínez 2008, Park et al. 2011a).

Como en los otros parámetros, cada especie necesita un rango determinado de $\mathrm{pH}$ que permita un crecimiento óptimo (Martínez 2008), siendo pH 8 el más indicado para especies dulceacuícolas (Park et al. 2011a). Por encima o debajo de éste, presentan un descenso en la productividad, que no solo afecta el crecimiento algal, sino también la capacidad de remover el nitrógeno en sistemas de tratamientos de aguas (Park et al. 2011a).

El pH puede controlarse con un sistema automatizado de inyección de $\mathrm{CO}_{2}$, o incluso, con adicción de ácido o base permitiendo además, suministrar $\mathrm{CO}_{2}$ necesario para cultivos de alta productividad (Berenguel et al. 2004, Martínez 2008, Sialve et al. 2009).

Las necesidades de $\mathrm{CO}_{2}$ pueden ser considerables, por lo que los costos asociados pueden ser también elevados para un cultivo comercial (Sialve et al. 2009, Mendoza et al. 2011, Acién et al. 2012), de ahí la importancia de buscar una forma económica de mitigar estos costos (Abatzoglou \& Boivin 2009, Acién et al. 2012). Así, se han realizado variados estudios sobre las capacidades de diferentes microalgas para la fijación de $\mathrm{CO}_{2}$ desde diversas fuentes gaseosas (Mann et al. 2009, Wang et al. 2010, Ho et al. 2011, 2012), considerándose altamente eficientes en este proceso (Ho et al. 2012). De esta manera, se ha sugerido la creación de cultivos comerciales para trabajar en conjunto con fuentes generadoras de $\mathrm{CO}_{2}$, como cogeneradores (Ho et al. 2011, 2012; Infante et al. 2012). También han sido utilizadas en la depuración de biogás a partir de digestión anaerobia, limpiando el gas resultante de $\mathrm{CO}_{2}$ y $\mathrm{H}_{2} \mathrm{~S}$ y elevando su capacidad calorífica (Mann et al. 2009, Sialve et al. 2009, Doušková et al. 2010, Park et al. 2011a, Rawat et al. 2011, Kao et al. 2012), aunque la literatura sobre la técnica aún es escasa (Abatzoglou \& Boivin 2009).

La adición de $\mathrm{CO}_{2}$ ha demostrado mejorar la productividad en HRAPs a escala experimental en tratamiento de aguas residuales, aumentando la productividad incluso a más del doble (Mann et al. 2009, Park et al. 2011b). Se trata de hecho, de una práctica estándar en todos los sistemas para producción de algas comerciales (Martínez 2008, Mendoza et al. 2011, Park et al. 2011b). 


\section{Nutrientes}

El nitrógeno es el nutriente más importante para las microalgas (después del carbono) y se incorpora como nitrato $\left(\mathrm{NO}_{3}^{-}\right)$o como amonio $\left(\mathrm{NH}_{4}^{+}\right)$(Grobbelaar 2004, Martínez 2008, Abdel-Raouf et al. 2012).

Es también un factor crítico para regular el contenido de lípidos de las microalgas (Park et al. 2011a). Típicamente, las microalgas tienen un contenido lipídico aproximadamente del 20\% (Benemann 2008 y Chisti 2008 en Park et al. 2011a), pero cuando el nitrógeno se convierte en el factor limitante del crecimiento, la acumulación de los niveles de lípidos aumenta en más de $40 \%$ (Park et al. 2011a, Ho et al. 2012, Ho et al. 2013). Sin embargo, usando la limitación de nitrógeno para estimular la acumulación de lípidos en las células de algas, a menudo reduce la producción de algas, lo que sugiere que las 2 condiciones, alto contenido en lípidos y alta productividad, pueden ser mutuamente excluyentes (Park et al. 2011a).

El fósforo es fundamental en muchos procesos celulares, tales como la formación de ácidos nucleicos y transferencia de energía (Grobbelaar 2004). Aunque el contenido en fósforo de las microalgas es menor al $1 \%$, su deficiencia en el medio de cultivo es una de las mayores limitaciones al crecimiento. En los medios de cultivo suele incorporarse en forma de $\mathrm{HPO}_{4}^{2-}$ o $\mathrm{HPO}_{4}^{-}$(Grobbelaar 2004, Martínez 2008).

\section{ZOOPLANCTON PASTOREADOR Y DEPREDADORES}

Los cultivos de microalgas son susceptibles al pastoreo por algunos grupos zooplantónicos, tales como cladóceros, rotíferos o nemátodos, sobre todo en sistemas abiertos (McGriff \& McKinney 1972, Park et al. 2011a). La presión por predación es tal, que en pocos días pueden reducir la productividad entre un 90 a 99\% (Oswald 1980 y Cauchie et al. 1995 en Park et al. 2011a).

Estas plagas se pueden tratar mediante medios físicos, tales como filtración, centrifugación, baja concentración de oxígeno disuelto (OD) / alta carga orgánica y tratamientos químicos, como aplicación de hormonas miméticas contra invertebrados, aumento de $\mathrm{pH}$ y concentración de amonio libre (Park et al. 2011a). Nurdogan \& Oswald (1995) mencionan que 20 microgramos por litro Dimilin ${ }^{\mathrm{TM}}$ (Diflubenzuron 25\%) en el estanque eliminó la población Daphnia en menos de una semana. Este producto afecta la formación de quitina en artrópodos, siendo altamente efectivo en su control, pero de manejo cuidadoso para evitar problemas ambientales y de salud. Por otro lado, muchos zooplancteres son capaces de sobrevivir largos periodos de baja OD, por lo que Park et al. (2011a) menciona que el ajuste del $\mathrm{pH}$ hasta un valor de 11 es quizás el método más práctico de control.

En la actualidad no hay tratamientos generales para el control de las infecciones por hongos (Park et al. 2011a).

\section{Oxígeno Disuelto}

La intensa fotosíntesis realizada durante el día en sistemas de cultivo puede aumentar los niveles de oxígeno disuelto a saturación $>200 \%$. Se cree que una elevada saturación podría afectar la productividad de algas. En 2001 Molina et al. (en Park et al. 2011a) determinó que a una saturación del $200 \%$ existe una reducción del $17 \%$ en la productividad, mientras que a $300 \%$ se reduce en un $25 \%$. Todavía se requiere más investigación para demostrar el efecto de altos niveles de oxígeno (Park et al. 2011a).

\section{Sistemas de Cultivo Para microalgas}

En la producción de organismos fotoautótrofos existen 2 diseños básicos (Borowitzka 1999, Contreras-Flores et al. 2003, Tredici 2004): cultivos abiertos, donde la biomasa está expuesta a las condiciones medioambientales; y cerrados, denominados fotobiorreactores o PBR (por sus siglas en inglés), con poco o ningún contacto con el medio externo (Contreras-Flores et al. 2003, Tredici 2004, Posten 2009).

Un sistema de cultivo es diseñado en base a diversos criterios: la biología de la(s) especie(s) a cultivar (Borowitzka 1999, Martínez 2008); forma del cultivo; requerimientos nutricionales, lumínicos y resistencia al estrés (Martínez 2008); relación de la superficie iluminada/ volumen del reactor que determina la velocidad de crecimiento (Contreras-Flores et al. 2003, Martínez 2008); orientación e inclinación; tipo de sistemas de mezcla y dispersión de gases; sistemas de limpieza y de regulación de la temperatura; transparencia y durabilidad del material (Borowitzka 1999, Martínez 2008); capacidad de escalado (Borowitzka 1999). Por último, son también importantes bajos costos de construcción y operación para fines comerciales (Martínez 2008).

La elección del tipo de sistema de cultivo es compleja, siendo importante determinar el tipo y valor del producto final desarrollado a partir de la biomasa, además de la disponibilidad de recursos hídricos y/o suelo (Borowitzka 1999, Martínez 2008). 


\section{Sistemas de CULTIVos abiertos}

Son los sistemas más comunes (Martínez 2008, Posten 2009). Comprenden tanto medios naturales, como lagunas y estanques, como artificiales con variedad de diseños. Por ejemplo, estanques circulares agitados mediante una paleta rotatoria usados en Japón, Taiwán e Indonesia para Chlorella (Borowitzka 1999, Martínez 2008, Posten 2009). Entre éstos, el más utilizado es el High Rate Algal Ponds (HRAP) o Raceway (Fig. 1A), excavación o estanque con una profundidad de entre 15 a $30 \mathrm{~cm}$, dividido por un muro central formando 2 canales (Posten 2009, Park et al. 2011b). El cultivo circula mediante paletas situadas en uno de los canales (Borowitzka 1999, Contreras-Flores et al. 2003, Martínez 2008, Posten 2009, Park et al. 2011b). Este sistema es de los más rentables, ya que puede ser utilizado para el tratamiento de aguas residuales de distintas fuentes (de Godos et al. 2009, Park et al. 2011b, Rawat et al. 2011, Abdel-Raouf et al. 2012), lo que disminuye los costos por requerimientos nutricionales

A

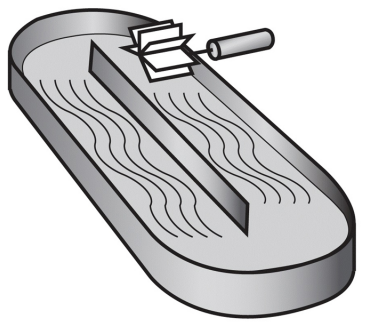

C

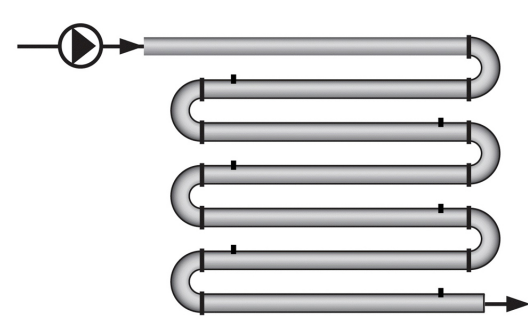

del cultivo (Abdel-Raouf et al. 2012, Acién et al. 2012), pudiendo alcanzar una concentración celular hasta $0,7 \mathrm{~g}$ $\mathrm{L}^{-1}$ (Contreras-Flores et al. 2003) y productividades por hectárea de hasta $50 \mathrm{t}$ año-1 (Rawat et al. 2011).

Las ventajas de los sistemas abiertos radican en su bajo costo y facilidad de construcción y operación, así como en la alta durabilidad (de Godos et al. 2009, Martínez 2008, Rawat et al. 2011, Abdel-Raouf et al. 2012). Como desventajas encontramos la baja accesibilidad de las células a la luz, la evaporación, la necesidad de grandes extensiones de terreno y exposición a contaminación por parte de organismos heterótrofos de rápido crecimiento y/o plancton pastoreador (Contreras-Flores et al. 2003, Martínez 2008, Posten 2009, Park et al. 2011b, Rawat et al. 2011). Por ello muchas producciones comerciales se reducen a especies que crecen en condiciones ambientales extremas, tales como $\mathrm{pH}$ y temperatura (Martínez 2008).

B
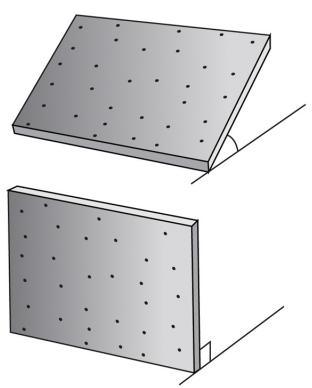

D

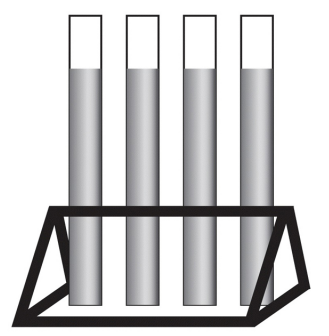

Figura 1. En los sistemas de cultivo, existe una gran variedad de diseños diferentes. Se pueden separar en 2 grandes grupos. Sistemas abiertos (A) y sistemas cerrados (B, C y D). a) cultivo tipo carrusel (High Rate Algal Ponds; HRAP), modificado desde Mendoza et al. (2011); b) fotobiorreactor en paneles, modificado desde modificado de Richmond (2004); c) y d) fotobiorreactores tubulares, modificados desde Posten (2009) y Kao et al. (2012), respectivamente / In culture systems, there are a variety of different designs. They can be separated into 2 groups. Open systems (A) and closed systems (B, C and D). a) High Rate Algal Ponds (HRAP), modified from Mendoza et al. (2009); b) flat photobioreactor modified from Richmond (2004); c) and d) tubular photobioreactor, modified from Posten (2009) and Kao et al. (2012), respectively 
Se cree que los sistemas abiertos, practicados desde hace más de medio siglo (Borowitzka 1999), han alcanzado el límite de desarrollo, restringiendo el avance en la biotecnología de microalgas (Contreras-Flores et al. 2003).

\section{Sistemas de CUltivo CerRados}

Las dificultades en los sistemas de cultivo abiertos han favorecido el desarrollo de los sistemas de cultivo cerrados (Borowitzka 1999, Contreras-Flores et al. 2003, Martínez 2008, Posten 2009). Éstos permiten un importante control de los parámetros, disminuyendo sustancialmente los problemas presentes en los sistemas abiertos (Martínez 2008, Posten 2009). Además, permiten realizar cultivos hiperconcentrados, ya sean mixtos o monoalgales, con valores superiores a $1,5 \mathrm{~g} \mathrm{~L}^{-1}$ (Lavoie \& de la Noüe 1985, Abdel-Raouf et al. 2012) pudiendo obtener Alta Densidad Celular (ADC) por sobre $3 \mathrm{~g} \mathrm{~L}^{-1}$ o Ultra Alta Densidad Celular (UADC) entre 15 a $80 \mathrm{~g} \mathrm{~L}^{-1}$ (ContrerasFlores et al. 2003).

Es necesario centrar los estudios de PBR para ficorremediación, ya que aún no es factible a gran escala debido a los grandes volúmenes necesarios (Martínez 2008, Rawat et al. 2011). Rawat et al. (2011) indica que, en pequeña escala, han probado ser altamente efectivos en tratamiento de aguas residuales, solamente con energía solar y a un costo mínimo de energía. Sin embargo, Posten (2009) indica la existencia del PBR de mayor tamaño a esa fecha en Klötze (Alemania), con un sistema de tubos de vidrio formando una red de cañerías con más de $500 \mathrm{~km}$ de longitud bajo invernadero.

Los diseños en fotobiorreactores son variados (Rawat et al. 2011): reactores planos o en placas (Fig. 1B), agitados mediante burbujeo; reactores tubulares (Fig. 1C y 1D), verticales u horizontales con agitación por burbujeo u otro; y reactores anulares, una variante de los reactores tubulares con una fuente lumínica interna (Martínez 2008, Posten 2009).

Menos masivos son los que tienen forma de domo, en bolsas plásticas (colgantes o formando una columna reforzada en malla), espirales y serpentines (Borowitzka 1999, Contreras-Flores et al. 2003, Martínez 2008, Posten 2009). Estos modelos tienen dificultad en el escalamiento (Borowitzka 1999), por lo que solo es aconsejado para estudios de laboratorio, pequeña escala y generación de biomasa para obtención de productos específicos.

Es importante considerar la velocidad del fluido, en especial, en los de tubo horizontal. A bajas velocidades $\left(<15 \mathrm{~cm} \mathrm{~s}^{-1}\right)$ es probable la sedimentación, crecimiento adosado a la pared del PBR e inhibición por alta concentración de oxígeno. Velocidades entre 30 a $50 \mathrm{~cm}$ $\mathrm{s}^{-1}$ son suficientes en la mayoría de los casos (ContrerasFlores et al. 2003).

\section{REDUCCIÓN DE COMPUESTOS CONTAMINANTES DESDE AGUAS RESIDUALES}

En el tratamiento de aguas residuales se busca eliminar la demanda bioquímica de oxígeno (DBO), sólidos suspendidos, nutrientes, coliformes y toxicidad (Dominic et al. 2009, Park et al. 2011a, Rawat et al. 2011, AbdelRaouf et al. 2012).

En condiciones apropiadas, las microalgas poseen una capacidad depuradora conocida como ficorremediación (Park et al. 2011a, Rawat et al. 2011, Prajapati et al. 2013), definida como el uso de macroalgas y/o microalgas para la eliminación o biotransformación de contaminantes, desde aguas residuales y desde un medio gaseoso (Olguín 2003, Dominic et al. 2009, Doušková et al. 2010, León \& Chaves 2010, González-López et al. 2011, Hongyang et al. 2011, Rawat et al. 2011, Abdel-Raouf et al. 2012, Infante et al. 2012; Prajapati et al. 2013; Maity et al. 2014).

Muchos factores están involucrados en el crecimiento y composición de especies de microalgas y sus capacidades depuradoras (Park et al. 2011a, Rawat et al. 2011), por lo que sus capacidades deben ser verificadas bajo las condiciones ambientales locales.

\section{Reducción de Demanda Biológica de OxíGENo (DBO) y Demanda Química de Oxígeno (DQO)}

Una elevada DBO implica una disminución del oxígeno disuelto del agua, pudiendo causar anaerobiosis y muerte de organismos. De ahí que su eliminación constituya un objetivo principal del tratamiento de aguas (Abdel-Raouf et al. 2012). La utilización de microalgas ha demostrado ser eficiente en la reducción de DBO y DQO (Li et al. 2011, Abdel-Raouf et al. 2012) proveyendo además de oxígeno a las bacterias aeróbicas que ayudan a la biotransformación (Abdel-Raouf et al. 2012).

Los valores de reducción de la DBO y DQO son variables (Tabla 1) puesto que dependen de varios factores ambientales (de Godos et al. 2009). En 1988, Colak \& Kaya (en Abdel-Raouf et al. 2012), en estudios sobre utilización de microalgas en aguas residuales urbanas, registraron valores moderados de remoción de DBO de $68,4 \%$. En 2008, similares resultados se observan por Hodaifa y colaboradores (en Brennan \& Owende 2010) con una remoción de $67,4 \%$ en cultivos de Scenedesmus 
obliquus (Turpin) Kützing 1833, alimentados con agua residual procedente de la producción de aceite de oliva. Mientras que León \& Chaves (2010) lograron una apreciable reducción de un $91,4 \%$ en tratamientos con lagunas de estabilización y microalgas.

En cuanto a la DQO, Hammouda et al. (1995) registraron para Scenedesmus sp. y Chlorella vulgaris Beyerinck [Beijerinck] 1890, una remoción de DQO de 89 y 91,7, respectivamente. Hongyang et al. (2011) al trabajar con C. pyrenoidosa Chick 1903 y aguas residuales del procesamiento de soja, obtuvieron una remoción de DQO de 80 y $84 \%$. Mientras que de Godos et al. (2009), al tratar aguas residuales desde porquerizas en HRAP, obtuvieron una remoción de $76 \pm 11 \%$. Colak \& Kaya, en 1988 (en Abdel-Raouf et al. 2012), observaron remociones bajas de $67,2 \%$. Por otro lado, Wang et al. (2010) obtuvieron las más bajas con 27,4 a $38,4 \%$ de remoción para aguas residuales desde lodos de digestión anaerobia de estiércol de vacunos. Por último, Li et al. (2011) registran valores de DQO de 90,3 y $90,8 \%$ en sus experimentos con Chlorella sp., concluyendo que las microalgas utilizaban rápidamente diferentes compuestos orgánicos como fuente de carbono, además del $\mathrm{CO}_{2}$.

\section{REMOCIÓN DE NITRÓGENO Y FÓSFORO}

Desde mediados del siglo XX se sabe que las microalgas son eficientes en la remoción de nitrógeno y fósforo en aguas residuales (Hammouda et al. 1995, Tam \& Wong 1996, Brennan \& Owende 2010, Park et al. 2011a, AbdelRaouf et al. 2012). Esto se debe a que son nutrientes esenciales en la formación de biomasa (Markou \& Georgakakis 2011), por lo que incorporan el amonio, nitrato y fósforo por absorción directa (Tam \& Wong 1996). Las principales formas en que se encuentra el nitrógeno en las aguas residuales son $\mathrm{NH}_{4}^{+}$(amonio), $\mathrm{NO}_{2}^{-}$(nitrito) y NO- (nitrato), mientras que el fosfato se presenta como $\mathrm{PO}_{4}^{3-}$ (ortofosfato) (Hammouda et al. 1995, Abdel-Raouf et al. 2012).

La descripción de la remoción de nutrientes en la literatura es variada (Tabla 1). A Chlorella vulgaris se le ha asignado una eficiencia de remoción de $86 \%$ para $\mathrm{N}$ inorgánico y 70\% para P inorgánico (Tam \& Wong 1996, Abdel-Raouf et al. 2012). Por otro lado, Wang et al. (2010), al trabajar con Chlorella sp., obtuvieron remociones de $100 \%$ para amonio, $75,7-82,5 \%$ para nitrito y $62,5-74,7 \%$ para fósforo. Li et al. (2011) utilizaron Chlorella sp. para el tratamiento de aguas residuales municipales altamente concentradas, con $93,9 \%$ para amonio, $89,1 \%$ para nitrógeno total y $80,9 \%$ para fósforo. Hongyang et al.
(2011) en el tratamiento de residuos agroindustriales y Chlorella pyrenoidosa, obtuvieron $88,8 \pm 1,0 \%, 89,1 \pm$ $0,6 \%$ y $70,3 \pm 11,4 \%$ para nitrógeno total, amonio y fósforo, respectivamente. Hammouda et al. (1995), en cultivos de laboratorio utilizando Chlorella sp. y Scenedesmus sp., obtuvieron remoción de $100 \%$ del nitrato, amonio y fósforo pasados 36, 42 y 48 días. En sistemas de cultivo mixto, McGriff \& McKinney (1972) obtuvieron reducciones de $92 \%$ para nitrógeno y $74 \%$ para fósforo en un pionero sistema de cultivo de laboratorio mixto que intercalaba luz/oscuridad, y donde las microalgas predominantes fueron Chlorella sp. y Nitzchia sp. Estos resultados concuerdan con lo observado por Nurdogan \& Oswald (1995) con remociones sobre $90 \%$ para ambos nutrientes en sistemas abiertos de cultivo mixto. Wang et al. (2010) lograron interesantes resultados con remociones de $100 \%$ para amonio, 75,7-82,5\% para nitrógeno total y $62,5-74,7 \%$ para fósforo total con Chlorella sp., depurando lodos diluidos procedentes de la digestión anaerobia de estiércol de ganado. Es importante considerar que altas concentraciones de nitrógeno en forma de amonio, no afectan significativamente el crecimiento de C. vulgaris (Tam \& Wong 1996).

También se ha demostrado que cultivos hiperconcentrados de algas (biomasa > 1,5 $\mathrm{g} \mathrm{L}^{-1}$ peso seco) son altamente efectivos, al acelerar la remoción de nutrientes en comparación a cultivos normales (Rawat et al. 2011). Es el caso de Oscillatoria sp. con $1,9 \mathrm{~g} \mathrm{~L}^{-1}$ peso seco (AbdelRaouf et al. 2012) y Scenedesmus obliquus con $2,6 \mathrm{~g} \mathrm{~L}^{-1}$ peso seco (Lavoie \& de la Noüe 1985).

La inmovilización de las microalgas ha sido reportado como una efectiva técnica que aumenta la remoción de nutrientes en comparación a la misma sin inmovilizar (Olguín 2003, Ruiz-Marín et al. 2010). Las microalgas inmovilizadas permitirían una mejor cosecha de la biomasa (Ruiz-Marín et al. 2010, Ruiz-Marín et al. 2011), pero no presentan diferencias nutritivas en la cantidad de proteínas y lípidos (Ruiz-Marín et al. 2010), ni en la actividad fotosintética entre células inmovilizadas y libres (Ruiz-Marín et al. 2011).

\section{REMOCIÓN DE METALES PESADOS}

La descarga de metales pesados a los sistemas de recolección de agua ha aumentado junto con la industrialización (Wilde \& Benemann 1993, Abdel-Raouf et al. 2012, Piotrowska-Niczyporuk et al. 2012). De ahí que sea esencial su eliminación, ya que son conocidos por su toxicidad, como por su papel como agentes cancerígenos (Srivastava \& Majumder 2008). 


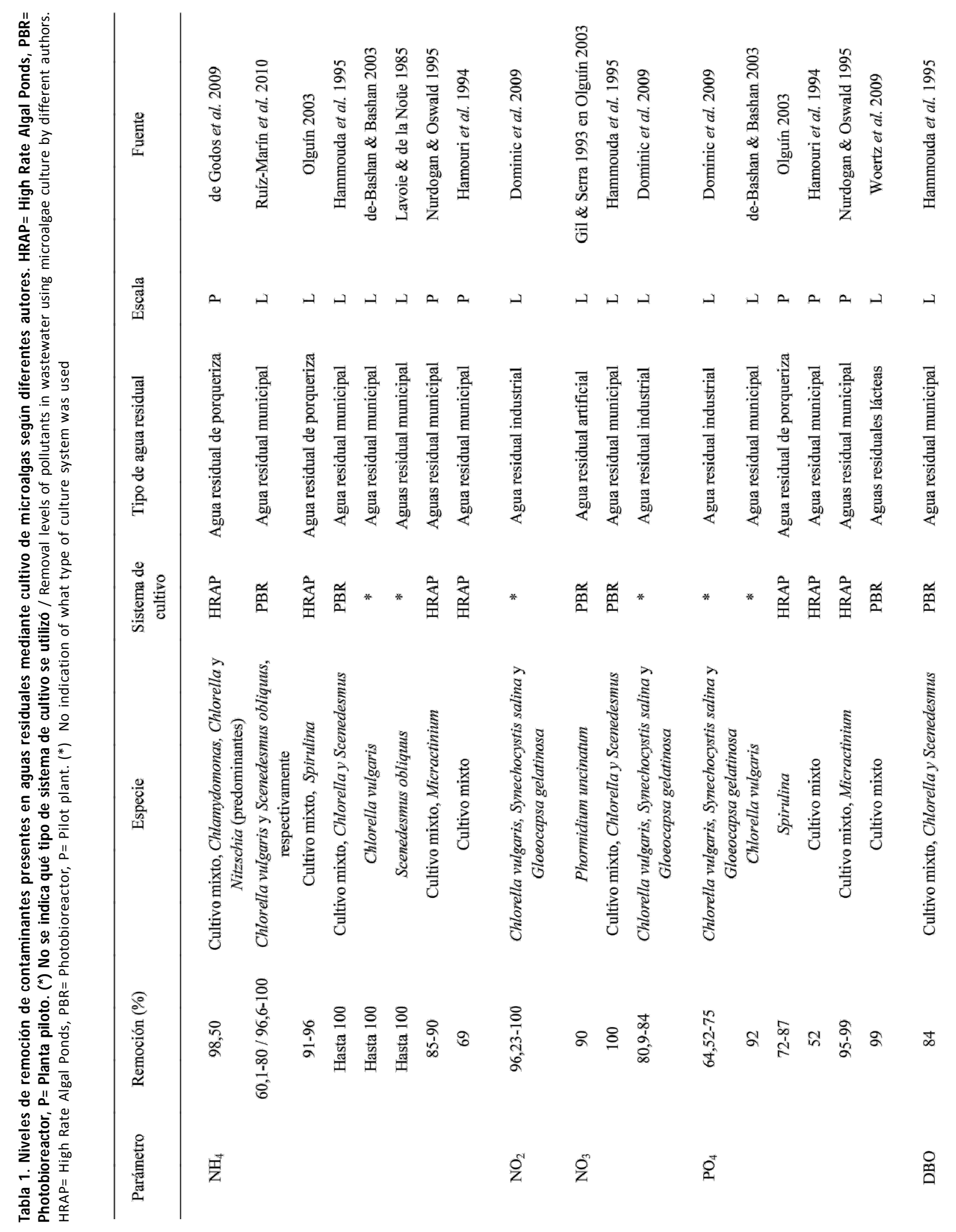

164 Hernández-Pérez \& Labbé

Microalgas, cultivo y beneficios 

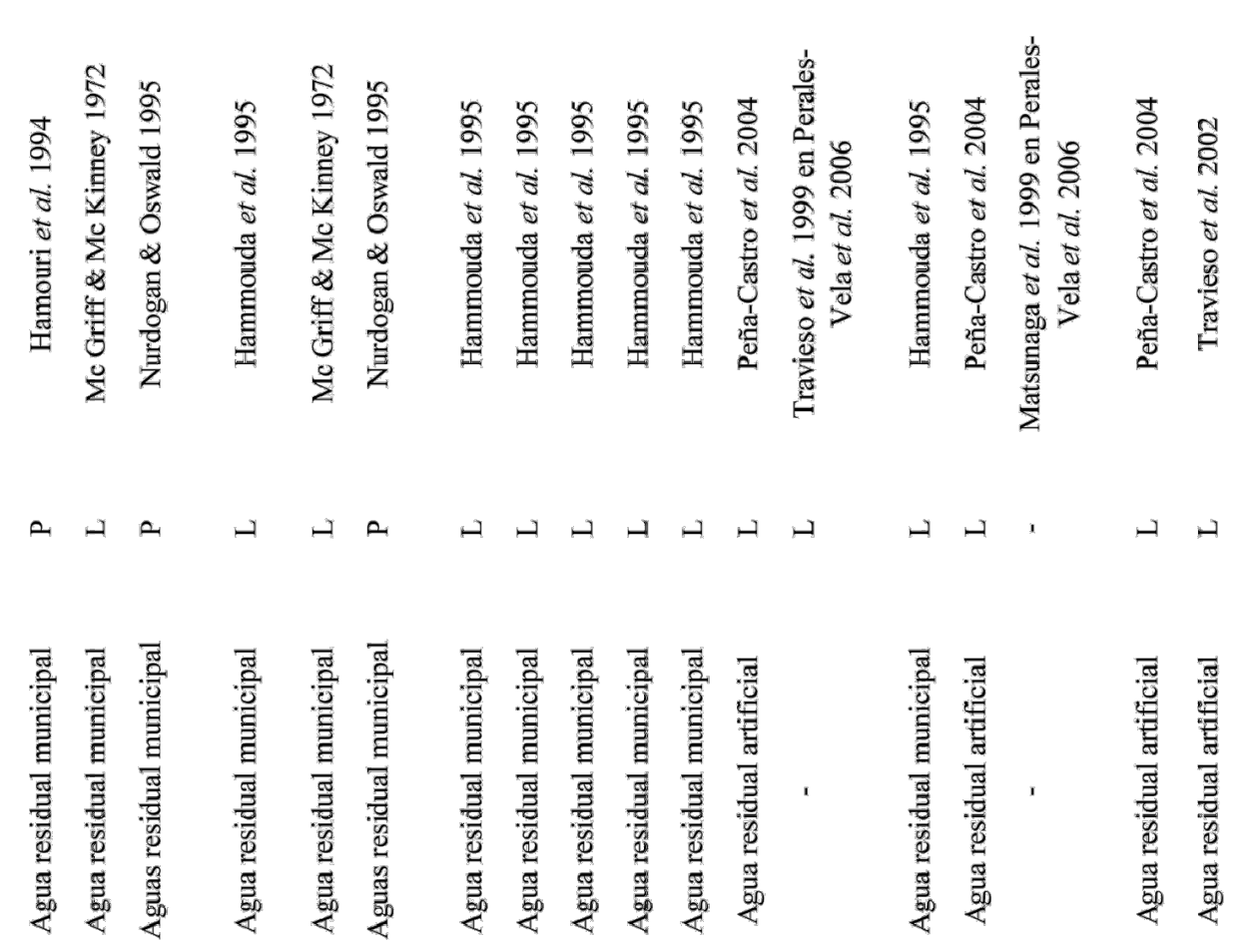

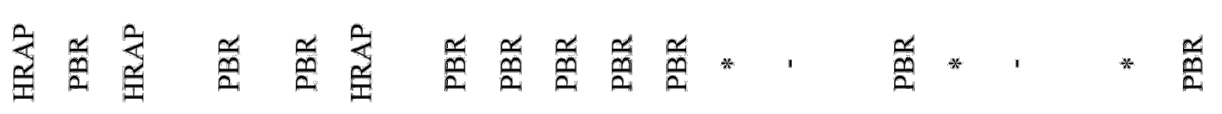
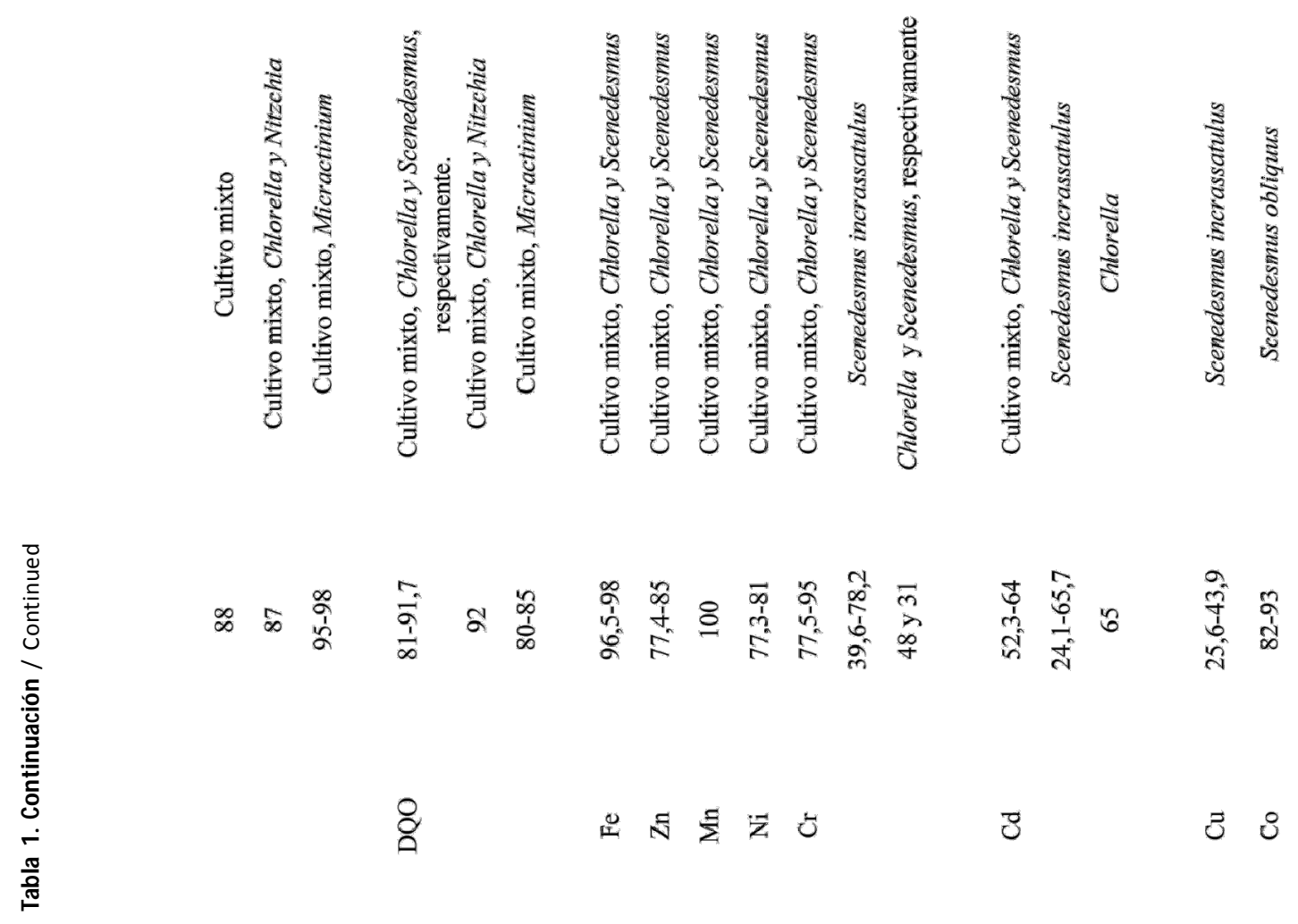

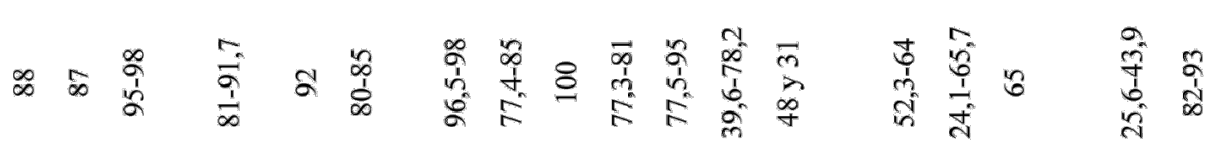

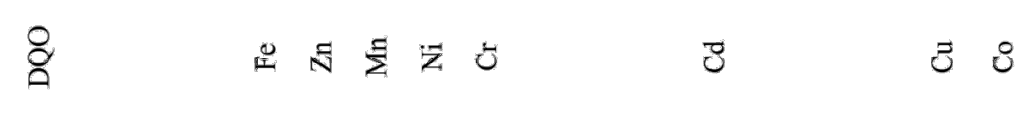


Muchas especies de microalgas han sido conocidas y estudiadas por su capacidad de retirar metales disueltos (Wilde \& Benemann 1993, Hammouda et al. 1995, Kaplan 2004; Srivastava \& Majumder 2008, Rawat et al. 2011, Piotrowska-Niczyporuk et al. 2012). Debido a su carga superficial negativa poseen una alta afinidad por los iones de metales pesados (Voltolina et al. 1998), introduciéndolos por adsorción, absorción y por formación de fitoquelatinas (Wilde \& Benemann 1993). Así, la utilización de microalgas en la remoción de metales pesados (Tabla 1), se considera un método económico con el resultado de un efluente de agua de alta calidad (Wilde \& Benemann 1993, Perales-Vela et al. 2006, Rawat et al. 2011, Abdel-Raouf et al. 2012, PiotrowskaNiczyporuk et al. 2012). En 1986, Gale registró una remoción del $99 \%$ de metales disueltos en agua de la minería por medio de microalgas (en Abdel-Raouf et al. 2012). Otras investigaciones demuestran que existe una amplia gama de especies de microalgas que absorben diferentes metales pesados (Wilde \& Benemann 1993, Seki et al. 2000, Kaplan 2004), soportando y adaptándose fisiológicamente al estrés por este tipo de contaminación (Perales-Vela et al. 2006, Piotrowska-Niczyporuk et al. 2012). Hammouda et al. (1995) determinaron una remoción del $100 \%$ utilizando Scenedesmus y Chlorella para Mn, 96,5-98\% para $\mathrm{Fe}, 77,4-85 \%$ para $\mathrm{Zn}, 77,5-95 \%$ para $\mathrm{Cr}$, $52,3-64 \%$ para $\mathrm{Cd}$, y $77,3-81 \%$ para $\mathrm{Ni}$. El género Scenedesmus es el más ampliamente estudiado con probadas capacidades para la remoción de $\mathrm{U}^{6+}, \mathrm{Cu}^{2+}, \mathrm{Cd}^{2+}$, $\mathrm{Zn}^{2+}, \mathrm{Co}^{2+}$ y Cr${ }^{6+}$ (Travieso et al. 2002, Peña-Castro et al. 2004, Perales-Vela 2006).

\section{REMoción de PATógenos}

Entre los organismos patógenos presentes en las aguas residuales están las bacterias, Escherichia coli, Salmonella y Shigella, virus y protozoos (Sebastian \& Nair 1984, Abdel-Raouf et al. 2012). Su presencia es considerada indicadora de contaminación fecal en los cuerpos de agua (Sebastian \& Nair 1984).

Un medio de cultivo favorable para las microalgas es desfavorable para microorganismos patógenos (Sebastian \& Nair 1984, Voltolina et al. 1998). El cambio de pH es la principal causa de este medio desfavorable, aumentando su efectividad si se mantiene por períodos de tiempo (Sebastian \& Nair 1984, Voltolina et al. 1998). La remoción de coliformes también puede ser variable dependiendo de la estación y ubicación del sistema de tratamiento (Hamouri et al. 1994). Se han reportado reducciones de coliformes fecales y Salmonella totales de $88,8 \%$, pero más frecuentemente entre 99,0 a 99,9\% (Sebastian \& Nair 1984, León \& Chaves 2010, Abdel-Raouf et al. 2012). Aun así, dadas las altísimas concentraciones de patógenos en los cuerpos de agua contaminadas, las concentraciones en los efluentes aún pueden estar por sobre los niveles permitidos (Sebastian \& Nair 1984). Ruiz- Marín et al. (2010) lograron un 95\% de remoción de coliformes fecales, sin embargo el rango resultante fue de 5,0 - 8,0 x $10^{4}$ MPNx100 $\mathrm{ml}^{-1}$, lo que hacía inviable su descarga a cuerpos naturales de aguas implicando una desinfección adicional.

\section{MÉtodos de COSECHA}

Uno de los principales problemas en el cultivo de microalgas es el método de recuperación o cosecha, ya sea para la producción de biomasa con valor comercial, para el tratamiento de aguas residuales y/o para la captura de $\mathrm{CO}_{2}$ (Lavoie \& de la Noüe 1985, Harun et al. 2010). De hecho, es considerado el paso más complejo y costoso del sistema, el cual depende en gran medida de las características del cultivo (Flotats et al. 2011, Rawat et al. 2011), tanto técnicas como biológicas. La selección del método de cosecha es de gran importancia para la economía de la producción de biocombustibles puesto que puede encarecer el costo total de la producción entre un 20-30\% (Flotats et al. 2011, Rawat et al. 2011, Acién et al. 2012, Prajapati et al. 2013). En la actualidad, las tecnologías de cosecha aún deben ser más eficientes y económicas para mejorar la viabilidad comercial del cultivo de microalgas, en especial, si está orientado a generación de biocombustibles (Chen et al. 2011, Acién et al. 2012).

Entre los métodos de recuperación de la biomasa se pueden encontrar centrifugación, sedimentación, filtración, flotación y floculación (Chen et al. 2011, Ho et al. 2011, Rawat et al. 2011, Abdel-Raouf et al. 2012).

La centrifugación es un excelente método para la separación de la biomasa, ya que es rápido y eficaz, pero económicamente inviable para la recolección a gran escala al ocupar mucha energía y requerir de equipos especializados (Harun et al. 2010, Chen et al. 2011, Mendoza et al. 2011). Esta técnica es factible cuando el valor de los productos resultantes es elevado (Ho et al. 2011).

La sedimentación por gravedad es un método común de recolección de biomasa (Chen et al. 2011, Park et al. 2011b, Abdel-Raouf et al. 2012). Pese a que es rudimentario, se perfila como el más eficiente método en relación a su efectividad y costo de ejecución (Ho et al. 2011, Park et al. 2011a). Además funciona para varios 
tipos de microalgas y es energéticamente eficiente (Rawat et al. 2011, Abdel-Raouf et al. 2012).

La filtración es un método comúnmente utilizado para la separación sólido-líquido (Rawat et al. 2011). Es considerado eficiente en la separación de células grandes, como Spirulina, pero no para especies de menor tamaño como es el caso de Chlorella y Scenedesmus (Harun et al. 2010, Chen et al. 2011, Ho et al. 2011, Mendoza et al. 2011, Rawat et al. 2011). Estos métodos tienden a ser costosos en energía, con reemplazos frecuentes de membrana y bombeo continuo de la biomasa (Rawat et al. 2011). A pesar de ello, se continúa avanzado en el mejoramiento técnico del proceso (Harun et al. 2010, Mendoza et al. 2011, Chen et al. 2011).

La flotación de la biomasa de algas ha probado ser un método prometedor en la recolección de algas unicelulares pequeñas y en ensayos a escala de laboratorio (Chen et al. 2011, Rawat et al. 2011). La técnica consiste en la adición de gases, como el ozono o aire, que interactúan con la carga de las paredes celulares, permitiendo la flotación de las microalgas y subsecuente separación. Pero la utilización de ozono es una desventaja al incrementar los costos (Rawat et al. 2011).

La floculación convencional trabaja por el mecanismo de dispersión de carga (Abdel-Raouf et al. 2012). La adición de sales metálicas (como aluminio) hace que interactúen con la carga negativa de las microalgas permitiendo la agregación. De este modo, se puede combinar con sedimentación, filtración o flotación, haciendo estos procesos más eficientes (Rawat et al. 2011). Sin embargo, los residuos de esta técnica hacen inviable su utilización cuando los productos necesiten cierto grado de pureza (Mendoza et al. 2011, Rawat et al. 2011), en especial para alimentación.

Las microalgas pueden generar un proceso de autofloculación que es la agregación espontánea que da como resultado la sedimentación de la microalga. Ésta puede ocurrir de forma natural o inducida por la limitación de carbono o de otros factores abióticos (Chen et al. 2011, Rawat et al. 2011).

\section{UTILIZACIÓN DE LA BIOMASA}

La biomasa algal tiene una amplia utilización que va desde biofertilizante a producción de biocombustibles, también para alimentación animal y humana, y para la obtención de productos biotecnológicos con uso en medicina, farmacia y/o cosmética (Gómez 2007, Brennan \& Owende 2010, Flotats et al. 2011).
Gómez (2007) indica los beneficios de los cultivos de microalgas en 3 puntos: a) 'el cultivo de microalgas es un sistema biológico eficiente de utilización de la energía solar para producir materia orgánica. Las microalgas crecen más rápido que las plantas terrestres y es posible obtener mayores rendimientos anuales de biomasa'; b) 'la composición bioquímica puede modificarse fácilmente variando las condiciones ambientales y/o la composición del medio de cultivo'; y c) 'bajo ciertas condiciones, muchas especies de microalgas pueden acumular en altas concentraciones compuestos de interés comercial, tales como proteínas, lípidos, almidón, glicerol, pigmentos naturales o biopolímeros'. El potencial de las microalgas es considerable, sobre todo si consideramos que existen varios millones de especies de algas y microalgas, en comparación con alrededor de 250.000 especies de plantas terrestres (Brennan \& Owende 2010).

\section{Producción de biocombustibles}

El mundo se enfrenta a futuros retos energéticos puesto que se ha indicado que las reservas de combustibles fósiles se agotarán en medio siglo (Harun et al. 2011, Rawat et al. 2011). Por este motivo es crucial explorar las fuentes renovables de energía para el futuro (Park et al. 2011b). Así, la utilización de la biomasa podría proporcionar aproximadamente $25 \%$ de las necesidades energéticas mundiales, siendo además una fuente de productos biotecnológicos, farmacéuticos y aditivos alimentarios (Rawat et al. 2011, Wang et al. 2012).

El uso de plantas comestibles para crear biocombustibles ha generado un gran debate que implica problemas de seguridad alimentaria. Las principales ventajas de las microalgas como fuente para la producción de biocombustible son: tasas de crecimiento altas y cortos tiempos de generación; requisitos mínimos de tierra (en comparación con otros sistemas de cultivo para producción de biocombustibles); alto contenido de lípidos y ácidos grasos; el uso de agua residual como fuente de nutrientes y/o captura de $\mathrm{CO}_{2}$ desde fuentes contaminante (Park et al. 2011b, Rawat et al. 2011, Maity et al. 2014).

Además, las productividades de microalgas son significativamente mayores que en plantas superiores (Arnold 2013, Maity et al. 2014) con proyecciones de alrededor $70 \mathrm{t} \mathrm{ha}^{-1} \mathrm{a}^{-1}$ (peso de cenizas) en comparación a cultivos terrestres con $3 \mathrm{t} \mathrm{ha}^{-1} \mathrm{a}^{-1}$ para la soja, $9 \mathrm{t} \mathrm{ha}^{-1} \mathrm{a}^{-1}$ para el maíz y 10-13 $\mathrm{t} \mathrm{ha}^{-1} \mathrm{a}^{-1}$ de álamos híbridos (Perlack et al. 2005 en Abdel-Raouf et al. 2012). También, el cultivo de microalgas no requiere de tierra cultivable y agua dulce, 
sino que puede llevarse a cabo en estanques poco profundos en suelos de capa dura, con agua salina o salobre (Sialve et. al. 2009). Así, la producción de biodiesel desde microalgas, podría alcanzar hasta $58.700 \mathrm{~L}$ aceite $\mathrm{ha}^{-1}$, que es una o 2 magnitudes mayor que la de cualquier otro cultivo energético (Chen et al. 2011).

En general, la producción de biocombustibles se puede clasificar en 2 grandes grupos: por conversión termoquímica y por conversión bioquímica. El primer grupo consiste en la descomposición térmica de la biomasa para producir productos combustibles e involucra las técnicas de: gasificación para producción de gas de síntesis; licuefacción termoquímica para producción de bioaceite; pirólisis para bioaceite, gas de síntesis y carbón vegetal; y combustión directa para producción de electricidad. La conversión bioquímica en cambio consiste en la degradación biológica de la biomasa a través de reacciones químicas y biológicas asociadas a procesos metabólicos de microorganismos, e involucra las técnicas de digestión anaerobia para producción de biogás; fermentación alcohólica para producir etanol; y la producción fotobiológica para producción de hidrógeno (Brennan \& Owende 2010, Harun et al. 2011, Rawat et al. 2011, Abdel-Raouf et al. 2012, Ho et al. 2013, Wang et al. 2012, Prajapati et al. 2013).

La generación de biodiesel desde microalgas, a partir de sus aceites (Sialve et al. 2009, Wang et al. 2010, Li et al. 2011) se presenta como una solución eficiente ante los problemas medioambientales de la actualidad y la probable escasez de petróleo en un futuro cercano (Zeng et al. 2011, Wang et al. 2012), debido a sus altas tasas de crecimiento y de producción de lípidos transesterificables (Flotats et al. 2011).

En comparación con el diesel de petróleo, el diesel de microalgas tiene una reducción de hasta un $78 \%$ en emisión de $\mathrm{CO}_{2}$ (Brennan \& Owende 2010). Además es económica y tecnológicamente mejor que la producción de biodiesel a partir de muchas especies vegetales terrestres por requerir menor superficie terrestre para obtener mayor biomasa (Hou et al. 2011, Wang et al. 2012).

\section{Alimentación y Salud humana}

El consumo humano de microalgas se limita a pocas especies debido al estricto control sobre seguridad alimentaria, factores comerciales, demanda del mercado y preparación específica. Los 3 géneros más destacados son Chlorella, Spirulina y Dunaliella, que se comercializan como suplementos alimentarios (Morris et al. 1999, Spolaore et al. 2006, Brennan \& Owende 2010, Mendoza et al. 2011).

También algunas especies se comercializan por su valor medicinal, como Chlorella que protege contra la insuficiencia renal y promoción del crecimiento de Lactobacillus intestinales. Además favorece la disminución en concentraciones de colesterol sanguíneo. En cambio Dunaliella se comercializa por su contenido de $\beta$-caroteno (Morris et al. 1999, Spolaore et al. 2006, Brennan \& Owende 2010, Mendoza et al. 2011). Se ha logrado determinar además, que una dieta con Chlorella aumenta el nivel de antioxidantes en fumadores, conformándola como un suplemento que favorece la salud (Lee et al. 2010). Por otro lado, los poli y monosacáridos de $C$. pyrenoidosa son una fuente altamente potencial para la creación de fármacos contra tumores (Sheng et al. 2007). Incluidos en una dieta $(10 \%)$ se ha logrado determinar que disminuye la asimilación y previene la acumulación de dioxinas (Takekoshi et al. 2005), cantidad que no ha presentado toxicidad en roedores (Day et al. 2009).

\section{Alimentación animal}

El 30\% de la producción mundial de microalgas está destinada al consumo animal y, de hecho, el 50\% de la producción de Arthrospira es con este fin (Spolaore et al. 2006). Algunas especies de algas, tales como los géneros Chlorella, Scenedesmus y Spirulina, tienen aspectos beneficiosos para los animales, mejoran la respuesta inmune, la infertilidad, el control de peso, producen una piel más sana y un pelo brillante (Spolaore et al. 2006, Brennan \& Owende 2010, Mendoza et al. 2011). Se utilizan en la alimentación de gatos, perros, peces de acuario, aves ornamentales, caballos, vacas y toros reproductores (Spolaore et al. 2006, Harun et al. 2010). Sin embargo, no se recomienda la alimentación prolongada sobre todo a altas concentraciones, en especial las dietas a base de cianobacterias (Spolaore $e t$ al. 2006, Brennan \& Owende 2010). A pesar de todo se ha determinado que por lo menos hasta un $10 \%$ de la dieta conformada por Chlorella no presenta toxicidad (Day et al. 2009).

En acuicultura, las microalgas son la principal fuente de alimentación de moluscos filtradores y estados larvarios de peces y crustáceos (Knauer \& Southgate 1997, Spolaore et al. 2006, Brennan \& Owende 2010, Harun et al. 2010, Mendoza et al. 2011). Los géneros más 
comunes para alimentación son Chlorella, Tetraselmis, Isochrysis, Pavlova, Phaeodactylum, Chaetoceros, Nannochloropsis, Skeletonema y Thalassiosira, que son consumidas principalmente vivas (Hammouda et al. 1995, Knauer \& Southgate 1997, Spolaore et al. 2006).

\section{Microalgas como biofertilizante}

Otra aplicación es la de biofertilizante (Abdel-Raouf et al. 2012). Algunas técnicas de conversión (especialmente la pirólisis) producen carbón vegetal, llamado Biochar o Biocarbón, que posee un alto potencial como fertilizante en la agricultura (Brennan \& Owende 2010).

\section{ÁcIDOS GRASOS POLIINSATURADOS}

Los ácidos poliinsaturados (PUFAs por sus siglas en inglés) son esenciales en el desarrollo y fisiología humana, además de tener un rol importante en la reducción del riesgo de enfermedades cardiovasculares. Actualmente, las fuentes más importantes de estos compuestos son los peces, donde la utilización de sus aceites es limitada debido al riesgo de acumulación de toxinas en el pez, olor y sabor a pescado, pobre estabilidad oxidativa y mezcla entre diferentes tipos de ácidos grasos (Spolaore et al. 2006, Brennan \& Owende 2010, Harun et al. 2010, Mendoza et al. 2011). Es destacable que de hecho, los PUFAs se encuentran en los peces debido a la alimentación de microalgas, por lo que es lógico considerar a las microalgas como potenciales fuentes de estos componentes esenciales (Spolaore et al. 2006). Las microalgas son una fuente importante de estos ácidos aunque, actualmente, el ácido docosahexaenoico (DHA) es el único disponible comercialmente (Spolaore et al. 2006, Brennan \& Owende 2010, Mendoza et al. 2011). Cherng \& Shih (2005) en estudios en ratones y hámsteres, determinaron que la alimentación con Chlorella era beneficiosa para estos animales, disminuyendo la cantidad de triglicéridos, colesterol total y colesterol LDL, previniendo hiperlipidemia y arteriosclerosis.

\section{Pigmentos Carotenoides y Ficobiliproteínas}

De los más de 400 tipos de los pigmentos carotenoides, solo unos pocos son comercializados: $\beta$-caroteno, astaxantina y, en menor importancia, luteína, zeaxantina y bixina. El uso más importante de éstos es como colorante natural para alimentos y como aditivo para animales y en cosmética. La relevancia nutricional y terapéutica de algunos carotenoides radica en su capacidad de actuar como provitamina $\mathrm{A}$, convirtiéndose en vitamina $\mathrm{A}$.
Además, poseen propiedades antiinflamatorias y se les ha atribuido propiedades anticancerígenas, aunque esto último aún está en investigación (Spolaore et al. 2006, Rosales et al. 2007, Mendoza et al. 2011).

Los mayores productores comerciales de ficobiliproteínas (ficoeritrina y ficocianina) son la cianobacteria Arthospira y la rodophyta Porphyiridium. La principal aplicación de estos compuestos es como colorante natural, pero han aumentado las investigaciones sobre sus propiedades en la salud humana ampliando su consumo al mercado farmacológico (Spolaore et al. 2006).

\section{Cosmética}

Algunas especies de microalgas son utilizadas en la industria de la cosmética para el cuidado de la piel, tales como Arthospira y Chlorella (i.e., cremas antiedad, productos refrescantes o regenerantes de la piel, emolientes y antiirritantes). Extractos ricos en proteínas de Arthospira reparan los signos prematuros de envejecimiento de la piel, ejercen un efecto tensor y previenen la formación de estrías. Extractos de $C$. vulgaris estimulan la síntesis de colágeno, apoyando la regeneración de tejidos y la reducción de arrugas, mientras que Nannochloropsis oculata posee excelentes propiedades para el estiramiento de la piel (Spolaore et al. 2006).

\section{Conclusiones}

Las investigaciones sobre sistemas de cultivo son bastante profundas, pero se necesita ahondar en la implementación a escala piloto en ficorremediación para producción de biomasa, más que repetir modelos en laboratorio. Ya existen cultivos a gran escala y el uso de microalgas en ficorremediación ha demostrado ser efectiva y eficiente disminuyendo los costos de producción. Por lo cual, la difusión de esta tecnología fuera del ámbito científico es fundamental para continuar su desarrollo. Es importante considerar que existen ciertas limitaciones a usos combinados. Por ejemplo, es poco probable que las leyes existentes en la mayoría de los países del orbe permitan la utilización de aguas residuales para cultivos orientados a la alimentación humana, por muy efectivo que sea el tratamiento y limpieza de estas.

El uso de suelos es un factor importante a la hora de determinar la localización de la planta de cultivo. Las instalaciones a gran escala necesitan extensas áreas de terreno (en especial los sistemas abiertos), por lo que es aconsejable que su localización no posea un elevado valor 
económico y/o ambiental (sectores no aptos para la agronomía u otras actividades comerciales). Al utilizar estos terrenos con bajo o nulo interés comercial, se genera un valor agregado (económico, social y ambiental) en la obtención de biomasa a partir de microalgas. Por ejemplo, se podrían emplazar en zonas desérticas que de otra manera, no serían utilizadas en ninguna otra actividad.

Es destacable que el uso de suelos adquiere una elevada importancia en la generación de biocombustibles. Actualmente existe un elevado debate ético sobre este tema dado que la mayor parte de la materia prima utilizada para la generación de biocombustibles procede de productos agrícolas utilizados en la alimentación humana (como la soja o la caña de azúcar), y obviamente, usando tierras fértiles para su crecimiento. La utilización de microalgas para generación de biocombustibles evitaría este debate, teniendo además la ventaja de ser un cultivo más productivo por unidad de espacio que los tradicionalmente usados como fuente de materia prima.

Considerando el potencial de las microalgas en ficorremediación, más los múltiples usos que se le da a su biomasa, se sugiere que su cultivo es económico y ambientalmente atractivo. A nuestra consideración, la combinación de ficorremediación de aguas residuales y/ o $\mathrm{CO}_{2}$ y generación de biomasa para generación de biocombustibles serían los usos más interesantes de desarrollar. Sin embargo, más estudios deben ser realizados al respecto, con especial énfasis en sistemas de cosechas más eficientes y a menores costos, evaluaciones económicas para los distintos usos de la biomasa, y en el caso de la generación de biocombustibles, comparar técnica y económicamente respecto a la gran variedad de otras fuentes tales como caña de azúcar y soja.

\section{LITERATURA CITADA}

Abatzoglou N \& S Boivin. 2009. A review of biogas purification processes. Biofuels, Bioproducts \& Biorefining 3: 42-71.

Abdel-Raouf N, AA Al-Homaidan \& IBM Ibraheem. 2012. Microalgae and wastewater treatment. Saudi Journal of Biological Sciences 19: 257-275.

Abeliovich A. 2004. Water pollution and bioremediation by microalgae: Water purification: Algae in wastewater oxidation ponds. In: Richmond A(ed). Handbook of microalgal culture: Biotechnology and applied phycology, pp. 430-438. Blackwell Science, Iowa.

Acién FG, JM Fernández, JJ Magán \& E Molina. 2012. Production cost of a real microalgae production plant and strategies to reduce it. Biotechnology Advances 30: 1344-1353.
Arnold M. 2013. Sustainable algal biomass products by cultivation in waste water flows. VTT Technology 147: 184. Technical Research Centre of Finland, Espoo.

Berenguel M, F Rodríguez, FG Acién \& JL García. 2004. Model predictive control of $\mathrm{pH}$ in tubular photobioreactors. Journal of Process Control 14: 377-387.

Borowitzka MA. 1999. Commercial production of microalgae: ponds, tanks, tubes and fermenters. Journal of Biotechnology 70: 313-332.

Brennan L \& P Owende. 2010. Biofuels from microalgae-A review of technologies for production, processing, and extractions of biofuels and co-products. Renewable and Sustainable Energy Reviews 14: 557-577.

Chen C-Y, K-L Yeh, R Aisyah, D-J Lee, J-S Chang. 2011. Cultivation, photobioreactor design and harvesting of microalgae for biodiesel production: A critical review. Bioresource Technology 102: 71-81.

Cherng J-Y \& M-F Shih. 2005. Preventing dyslipidemia by Chlorella pyrenoidosa in rats and hamsters after chronic high fat diet treatment. Life Sciences 76: 3001-3013.

Chiu S-Y, C-Y Kao, T-T Huang, C-J Lin, S-C Ong, C-D Chen, J-S Chang \& C-S Lin. 2011. Microalgal biomass production and on-site bioremediation of carbon dioxide, nitrogen oxide and sulfur dioxide from flue gas using Chlorella sp. cultures. Bioresource Technology 102: 91359142.

Contreras-Flores C, J Peña-Castro, L Flores-Cotera \& $\mathbf{R}$ Cañizares-Villanueva. 2003. Avances en el diseño conceptual de fotobiorreactores para el cultivo de microalgas. Interciencia 28(8): 450-456.

Day AG, D Brinkmann, S Franklin, K Espina, G Rudenko, A Roberts \& KS Howse. 2009. Safety evaluation of a high-lipid algal biomass from Chlorella protothecoides. Regulatory Toxicology and Pharmacology 55: 166-180.

De Godos I, S Blanco, PA García-Encina, E Becares \& R Muñoz. 2009. Long-term operation of high rate algal ponds for the bioremediation of piggery wastewaters at high loading rates. Bioresource Technology 100: 4332-4339.

Dominic VJ, S Murali \& MC Nisha. 2009. Phycoremediation efficiency of three algae Chlorella vulgaris, Synechocystis salina and Gloeocapsa gelatinosa. Academic Review 16(12): $138-146$.

Doušková I, F Kaštánek, Y Maléterová, P Kaštánek, J Doucha \& V Zachleder. 2010. Utilization of distillery stillage for energy generation and concurrent production of valuable microalgal biomass in the sequence: Biogascogeneration-microalgae-products. Energy Conversion and Management 51: 606-611.

Flotats X, HL Foged, AB Blasi, J Palatsi, A Magri \& KM Schelde. 2011. Manure Processing Activities in Europe Project reference: ENV.B.1/ETU/2010/0007 Manure Processing Technologies. Technical Report 2, European Commission, Directorate-General Environment, 184 pp. 
<http://ec.europa.eu/environment/water/water-nitrates/pdf/ manure_processing.zip>

Gómez L. 2007. Microalgas: Aspectos ecológicos y biotecnológicos. Revista Cubana de Química 19(2): 3-20.

González-López CV, FG Acién, JM Fernández-Sevilla \& E Molina. 2011. Uso de microalgas como alternativa a las tecnologías disponibles de mitigación de emisiones antropogénicas de $\mathrm{CO}_{2}$. Revista Latinoamericana de Biotecnología Ambiental Algal 2(2): 93-106.

Grobbelaar JU. 2004. Algal nutrition: mineral nutrition. In: Richmond A (ed). Handbook of microalgal culture: Biotechnology and applied phycology, pp. 97-115. Blackwell Science, Iowa.

Hammouda O, A Gaber \& N Abdel-Raouf. 1995. Microalgae and wastewater treatment. Ecotoxicology and Environmental Safety 31: 205-210.

Hamouri B El, K Khallayoune, K Bouzoubaa, N Rhallabi \& M Chalabi. 1994. High-rate Algal Ponds performances in faecal coliforms and helminth egg removals. Water Research 28(1): 171-174.

Harun R, M Singh, GM Forde \& MK Danquah. 2010. Bioprocess engineering of microalgae to produce a variety of consumer products. Renewable and Sustainable Energy Reviews 14: 1037-1047.

Harun R, M Davidson, M Doyle, R Gopiraj, M Danquah \& G Forde. 2011. Technoeconomic analysis of an integrated microalgae photobioreactor, biodiesel and biogas production facility. Biomass and Bioenergy 35: 741-747.

Ho S-H, C-Y Chen, D-J Lee \& J-S Chang. 2011. Perspectives on microalgal $\mathrm{CO}_{2}$-emission mitigation systems - A review. Biotechnology Advances 29: 189-198.

Ho S-H, C-Y Chen \& J-S Chang. 2012. Effect of light intensity and nitrogen starvation on $\mathrm{CO} 2$ fixation and lipid/ carbohydrate production of an indigenous microalga Scenedesmus obliquus CNW-N. Bioresource Technology 113: 244-252.

Ho S-H, S-W Huang, C-Y Chen, T Hasunuma, A Kondo \& J-S Chang. 2013. Characterization and optimization of carbohydrate production from an indigenous microalga Chlorella vulgaris FSP-E. Bioresource Technology 135: 157-165.

Hongyang S, Z Yalei, Z Chunmin, Z Xuefei \& L Jinpeng. 2011. Cultivation of Chlorella pyrenoidosa in soybean processing wastewater. Bioresource Technology 102: 98849890.

Hou J, P Zhang, X Yuan \& Y Zheng. 2011. Life cycle assessment of biodiesel from soybean, jatropha and microalgae in China conditions. Renewable and Sustainable Energy Reviews 15: 5081-5091.

Infante C, E Angulo, A Zárate, JZ Florez, F Barrios \& C Zapata. 2012. Propagación de la microalga Chlorella sp. en cultivo por lote: cinética del crecimiento celular. Avances en Ciencias e Ingeniería 3(2): 159-164.
Kao C-Y, S-Y Chiu, T-T Huang, L Dai, L-K Hsu \& C-S Lin. 2012. Ability of a mutant strain of the microalga Chlorella sp. to capture carbon dioxide for biogas upgrading. Applied Energy 93: 176-183.

Kaplan D. 2004. Water pollution and bioremediation by microalgae: Absorption and adsorption of heavy metals by microalgae. In: Richmond A (ed). Handbook of microalgal culture: Biotechnology and applied phycology, pp. 439447. Blackwell Science, Iowa.

Knauer J \& PC Southgate. 1997. Growth and fatty acid composition of Pacific oyster (Crassostrea gigas) spat fed a spray-dried freshwater microalga (Spongiococcum excentricum) and microencapsulated lipids. Aquaculture 154: 293-303.

Lavoie A \& J de la Noüe. 1985. Hyperconcentrated cultures of Scenedesmus obliquus: Anew approach of wastewater biological tertiary treatment? Water Research 19(11): 1437-1442.

Lee SH, HJ Kang, H-J Lee, M-H Kang \& YK Park. 2010. Six-week supplementation with Chlorella has favorable impact on antioxidant status in Korean male smokers. Nutrition 26: 175-183.

León C \& D Chaves. 2010. Tratamiento de residual vacuno utilizando microalgas, la lenteja de agua Lemna aequinoctiales y un humedal subsuperficial en Costa Rica. Revista Latinoamericana de Biotecnología Ambiental Algal 1(2): 155-177.

Li Y, Y-F Chen, P Chen, M Min, W Zhou, B Martinez, J Zhu \& R Ruan. 2011. Characterization of a microalga Chlorella sp. well adapted to highly concentrated municipal wastewater for nutrient removal and biodiesel production. Bioresource Technology 102: 5138-5144.

Maity JP, J Bundschuh, C-Y Chen \& P Bhattacharya. 2014. Microalgae for third generation biofuel production, mitigation of greenhouse gas emissions and wastewater treatment: Present and future perspectives: A mini review. Energy. 〈http://dx.doi.org/10.1016/j.energy.2014.04.003>.

Mann G, M Schlegel, R Schumann \& A Sakalauskas. 2009. Biogas-conditioning with microalgae. Agronomy Research 7(1): 33-38.

Markou G \& D Georgakakis. 2011. Cultivation of filamentous cyanobacteria (blue-green algae) in agro-industrial wastes and wastewaters: A review. Applied Energy 88: 3389-3401.

Martínez L. 2008. Eliminación de $\mathrm{CO}_{2}$ con microalgas autóctonas. Tesis Doctoral, Instituto de Recursos Naturales, Universidad de León, León, 226 pp.

McGriff J \& RE McKinney. 1972. The removal of nutrients and organics by activated algae. Water Research 6: 1155-1164.

Mendoza H, A de la Jara \& E Portillo. 2011. Planta piloto de cultivo de microalgas: Desarrollo potencial de nuevas actividades económicas asociadas a la biotecnología en Canarias, 60 pp. Instituto Tecnológico de Canarias, Santa Cruz de Tenerife. 
Morris H, M Quintana, A Almarales \& L Hernández. 1999. Composición bioquímica y evaluación de la calidad proteica de la biomasa autotrófica de Chlorella vulgaris. Revista Cubana de Alimentación y Nutrición 13: 123-128.

Nurdogan Y \& W Oswald. 1995. Enhanced nutrient removal in high-rate ponds. Water Science and Technology 31(12): 33-43.

Olguín EJ. 2003. Phycoremediation: key issues for costeffective nutrient removal processes. Biotechnology Advances 22: 81-91.

Park J, R Craggs \& A Shilton. 2011a. Wastewater treatment high rate algal ponds for biofuel production. Bioresource Technology 102: 35-42.

Park J, R Craggs \& A Shilton. 2011b. Recycling algae to improve species control and harvest efficiency from a high rate algal pond. Water Research 45: 6637-6649.

Peña-Castro JM, F Martínez-Jerónimo, F Esparza-García \& RO Cañizares-Villanueva. 2004. Heavy metals removal by the microalga Scenedesmus incrassatulus in continuous cultures. Bioresource Technology 94: 219-222.

Perales-Vela HV, JM Peña-Castro \& RO CañizaresVillanueva. 2006. Heavy metal detoxification in eukaryotic microalgae. Chemosphere 64: 1-10.

Piotrowska-Niczyporuk A, A Bajguz, E Zambrzycka \& B Godlewska-Zylkiewicz. 2012. Phytohorones as regulators of heavy metal biosorption and toxicity in green alga Chlorella vulgaris. Plant Physiology and Biochemistry 52: 52-65.

Posten C. 2009. Design principles of photo-bioreactors for cultivation of microalgae. Engineering in Life Science 9(3): 165-177.

Prajapati SK, P Kaushik, A Malik \& VK Vijay. 2013. Phycoremediation coupled production of algal biomass, harvesting and anaerobic digestion: Possibilities and challenges. Biotechnology Advances 31: 1408-1425.

Rawat I, R Ranjith-Kumar, T Mutanda \& F Bux. 2011. Dual role of microalgae: Phycoremediation of domestic wastewater and biomass production for sustainable biofuels production. Applied Energy 88: 3411-3424.

Richmond A. 2004. Biological principles of mass cultivation. In: Richmond A (ed). Handbook of microalgal culture: Biotechnology and applied phycology, pp. 125-177. Blackwell Science, Iowa.

Rosales N, J Bermúdez, R Moronta \& E Morales. 2007. Gallinaza: un residual avícola como fuente alternativa de nutrientes para producción de biomasa microalgal. Revista Colombiana de Biotecnología 9(1): 41-48.

Ruiz-Marín A, LG Mendoza-Espinosa \& T Stephenson. 2010. Growth and nutrient removal in free and immobilized green algae in batch and semi-continuous cultures treating real wastewater. Bioresource Technology 101: 58-64.
Ruiz-Marín A, LG Mendoza-Espinosa \& M del P SánchezSaavedra. 2011. Photosynthetic characteristics and growth of alginate-immobilized Scenedesmus obliquus. Agrociencia 45: 303-313.

Sebastian S \& KVK Nair. 1984. Total removal of coliforms and $E$. coli from domestic sewage by High-Rate Pond Mass culture of Scenedesmus obliquus. Environmental Pollution (Series A) 34: 197-206.

Seki H, A Suzuki \& Y Iburi. 2000. Biosorption of heavy metal ions to a marine microalga, Heterosigma akashiwo (Hada) Hada. Journal of Colloid and Interface Science 229: 196-198.

Sheng J, F Yu, Z Xin, L Zhao, X Zhu \& Q Hu. 2007. Preparation, identification and their antitumor activities in vitro of polysaccharides from Chlorella pyrenoidosa. Food Chemistry 105: 533-539.

Sialve B, N Bernet \& O Bernard. 2009. Anaerobic digestion of microalgae as a necessary step to make microalgal biodiesel sustainable. Biotechnology Advances 27(4): 409416.

Spolaore P, C Joannis-Cassan, E Duran \& A Isambert. 2006. Commercial Applications of Microalgae. Journal of Bioscience and Bioengineering 101(2): 87-96.

Srivastava NK \& CB Majumder. 2008. Novel biofiltration methods for the treatment of heavy metals from industrial wastewater. Journal of Hazardous Materials 15: 1-8.

Stager C. 2012. El futuro profundo: Los próximos 100.000 años de vida en la Tierra, 358 pp. Editorial Crítica, Barcelona.

Takekoshi H, G Suzuki, H Chubachi \& M Nakano. 2005. Effect of Chlorella pyrenoidosa on fecal excretion and liver accumulation of polychlorinated dibenzo-p-dioxin in mice. Chemosphere 59: 297-304.

Tam NFY \& YS Wong. 1996. Effect of ammonia concentrations on growth of Chlorella vulgaris and nitrogen removal from media. Bioresource Technology 57: 45-50.

Travieso L, A Pellón, F Benítez, E Sánchez, R Borja, N O'Farrill \& P Weiland. 2002. BIOALGA reactor: preliminary studies for heavy metals removal. Biochemical Engineering Journal 12: 87-91.

Tredici MR. 2004. Mass production of microalgae: Photobioreactors. In: Richmond A (ed). Handbook of microalgal culture: Biotechnology and applied phycology, pp. 178-214. Blackwell Science, Iowa.

Voltolina D, B Cordero, M Nieves \& LP Soto. 1998. Growth of Scenedesmus sp. in artificial wastewater. Bioresource Technology 68: 265-268.

Wang L, Y Li, P Chen, M Min, Y Chen, J Zhu \& RR Ruan. 2010. Anaerobic digested dairy manure as a nutrient supplement for cultivation of oil-rich green microalgae Chlorella sp. Bioresource Technology 101: 2623-2628. 
Wang Y, T Chen \& S Qin. 2012. Heterotrophic cultivation of Chlorella kessleri for fatty acids production by carbon and nitrogen supplements. Biomass and Bioenergy 47: $402-$ 409.

Wilde EW \& JR Benemann. 1993. Bioremoval of heavy metals by the use of microalgae. Biotechnology Advances 11: 781812.
Zeng X, MK Danquah, X Dong-Chen \& Y Lu. 2011. Microalgae bioengineering: From $\mathrm{CO}_{2}$ fixation to biofuel production. Renewable and Sustainable Energy Reviews 15:3252-3260.

Recibido el 11 de septiembre de 2013 y aceptado el 2 de junio de 2014

Editor Asociado : Pilar Muñoz M. 